\section{Hodgkin-Lymphom: Konkurrenz für ABVD?}

\begin{abstract}
Seit langem ist das ABVD-Schema in Nordamerika Therapiestandard bei Patienten mit fortgeschrittenem Hodgkin-Lymphom. Eine Phase-III-Intergroup-Studie sollte klären, ob das Stanford-V-Regime hier Vorteile bietet.
\end{abstract}

$\mathrm{V}$ ier Studiengruppen aus den USA und Kanada, koordiniert von der Eastern Cooperative Oncology Group (ECOG), nahmen in ihre Studie $854 \mathrm{~Pa}$ tienten mit einem M. Hodgkin in den Stadien III/IV oder mit großer Tumormasse im Bereich des Mediastinums auf. Sie erhielten randomisiert entweder ABVD (Doxorubicin, Bleomycin, Vinblastin, Dacarbazin) in sechs bis acht $\mathrm{Zy}$ klen oder das Regime Stanford V (Doxorubicin, Vinblastin, Mechlorethamin, Etoposid, Vincristin, Bleomycin, Prednison) über zwölf Wochen.

Im ABVD-Arm wurden $41 \%$ der Patienten bestrahlt (nur bei mediastinaler großer Tumormasse), von den Teilnehmern im Stanford-V-Arm 75\% (hier auch auf alle Herde über $5 \mathrm{~cm}$ oder bei
Bedarf die Milz). Stratifiziert wurde nach Befall (Stadien I-IIA/B mit großem mediastinalem Tumor vs. Stadium IIIIV) und nach dem International Prognostic Factors Project Score (0-2 vs. 3-7 Punkte).

Bei den kompletten Remissionen (inklusive klinischer Remission) ergab sich mit $73 \%$ für ABVD und $69 \%$ für Stanford V kein signifikanter Unterschied (Tab. 1). Gleiches galt für das 5-JahresÜberleben ohne Therapieversagen mit $74 \%$ vs. $71 \%$ sowie für das Gesamtüberleben mit $88 \%$ in beiden Gruppen.

Fazit: Die großangelegte Studie zeigte keine Vorteile für Stanford V, ABVD bleibt in Nordamerika das StandardSchema bei fortgeschrittenem M. Hodg-

\begin{tabular}{|c|c|c|}
\hline \multicolumn{3}{|c|}{$\begin{array}{l}\text { Das Stanford V-Schema war } \\
\text { der ABVD-Therapie nicht überlegen }\end{array}$} \\
\hline Ansprechen & $\begin{array}{l}\text { ABVD-Arm } \\
(n=394)\end{array}$ & $\begin{array}{l}\text { Stanford V-Arm } \\
(\mathrm{n}=399)\end{array}$ \\
\hline CR und CCR & $72,7 \%$ & $68,7 \%$ \\
\hline PR & $7,6 \%$ & $7,5 \%$ \\
\hline SE & $8,4 \%$ & $10,5 \%$ \\
\hline Progression & $0,3 \%$ & $2,0 \%$ \\
\hline \multicolumn{3}{|c|}{$\begin{array}{l}\text { ABVD = Doxorubicin, Bleomycin, Vinblastin, Da- } \\
\text { carbazin; } C C R=\text { komplettes klinisches Ansprechen; } \\
C R=\text { komplettes Ansprechen; } P R=\text { partielle Remis- } \\
\text { sion, } S E=\text { stabile Erkrankung }\end{array}$} \\
\hline
\end{tabular}

kin. Die Deutsche Hodgkin-Studiengruppe verfolgt bekanntlch eine andere Strategie, bei der fortgeschrittene Stadien von Anfang an mit dem intensiveren BEACOPP-Regime behandelt werden.

Andreas Fischer

Gordon LI et al. Randomized phase III trial of $A B V D$ versus Stanford $V$ with or without radiation therapy in locally extensive and advanced-stage Hodgkin lymphoma: An intergroup study coordinated bei the Eastern Cooperative Oncology Group (E2496). J Clin Oncol. 2013;31(6):684-91.

\title{
Genexpressionsmuster sagt Sterberisiko vorher
}

\section{Noch immer sterben 10-15\% der Patienten mit fortgeschrittenem klassischem Hodgkin-Lymphom an ihrer Erkrankung. Lässt sich mithilfe eines Genexpressionsmusters das Sterberisiko vorherzusagen?}

ieße sich mithilfe eines Biomarkers — bereits zum Zeitpunkt der Diagnose zwischen Patienten mit niedrigem und hohem Sterberisiko differenzieren, könnte man dem Risiko entsprechend entweder eine Therapie mit geringen $\mathrm{Ne}$ benwirkungen und Langzeitfolgen oder eine dosisintensive Therapie auswählen. Der International Prognostic Score (IPS), entwickelt anhand von Daten aus den 1980er-Jahren, ist angesichts moderner Therapien nicht aussagekräftig genug.

Um Patienten mit fortgeschrittenem klassischem Hodgkin-Lymphom und erhöhtem Sterberisiko zuverlässig zu identifizieren, sollte in einer IntergroupStudie ein aussagekräftiger Prädiktor des Gesamtüberlebens anhand der Genexpression in Formalin-fixiertem, in Paraffin eingebettetem Gewebe von 290 Patienten entwickelt werden. In den vor der Behandlung entnommenen Biopsien wurden die Expressionslevel von 259 Genen bestimmt. 52 Gene waren signifikant mit dem Gesamtüberleben assoziiert. Schließlich wurden die Expressionslevel von 23 Genen für die Entwicklung eines Prädiktors verwendet, der zwischen Patienten mit niedrigem und hohem Risiko unterscheiden konnte.

Dieses Modell wurde in einer unabhängigen Kohorte von 78 Patienten getestet. Bei diesen Patienten war es zu Therapieversagen gekommen, ansonsten waren sie mit den Patienten eines bevölkerungsbasierten Registers vergleichbar. Anhand des Prädiktors konnte hier eine Population mit erhöhtem Sterberisiko identifiziert werden, die ein 5-JahresGesamtüberleben von $63 \%$ aufwies - gegenüber $92 \%$ in der NiedrigrisikoGruppe $(\mathrm{p}<0,001)$.
Das Sterberisiko der HochrisikoGruppe war damit fast siebenmal so hoch wie in der Niedrigrisiko-Gruppe (Hazard Ratio 6,7). Die Patienten der Hochrisiko-Gruppe waren im Allgemeinen älter, hatten öfter einen HochrisikoIPS, Epstein-Barr-Virus-positive Hodgkin-Reed-Sternberg-Zellen und keine noduläre Sklerose.

Insgesamt war das Genexpressionsmuster als Prädiktor für das Sterberisiko in multivariaten Analysen dem IPS und der CD68-Immunohistochemie überlegen.

Fazit: Durch die Analyse der Genexpression in vor Therapiebeginn entnommenen, eingefrorenen und in Paraffin eingebetteten Biopsien ist es möglich, diejenigen Patienten mit fortgeschrittenem klassischem Hodgkin-Lymphom zu identifizieren, die ein erhöhtes Sterberisiko haben.

Judith Neumaier

Scott DW et al. Gene expression-based model using formalin-fixed paraffin-embedded biopsies predicts overall survival in advancedstage classical Hodgkin lymphoma. J Clin Oncol. 2013;31(6):692-700. 